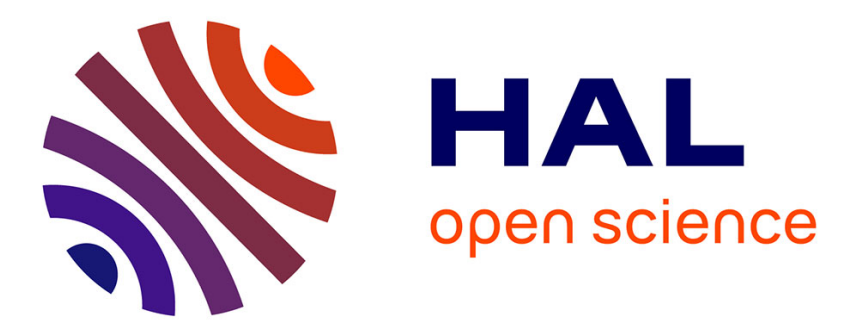

\title{
A motional narrowing diagnostic for E.S.R. lines a vibronic system
}

\author{
R. Englman
}

\section{To cite this version:}

R. Englman. A motional narrowing diagnostic for E.S.R. lines a vibronic system. Journal de Physique Lettres, 1976, 37 (10), pp.261-263. 10.1051/jphyslet:019760037010026100 . jpa-00231288

\section{HAL Id: jpa-00231288 https://hal.science/jpa-00231288}

Submitted on 1 Jan 1976

HAL is a multi-disciplinary open access archive for the deposit and dissemination of scientific research documents, whether they are published or not. The documents may come from teaching and research institutions in France or abroad, or from public or private research centers.
L'archive ouverte pluridisciplinaire HAL, est destinée au dépôt et à la diffusion de documents scientifiques de niveau recherche, publiés ou non, émanant des établissements d'enseignement et de recherche français ou étrangers, des laboratoires publics ou privés. 
Classification

Physics Abstracts

$7.160-8.632$

\title{
A MOTIONAL NARROWING DIAGNOSTIC FOR E.S.R. LINES A VIBRONIC SYSTEM
}

\author{
R. ENGLMAN $\left(^{*}\right)$
}

Laboratoire de Spectrométrie Physique, Université Scientifique et Médicale, B.P. 53, 38041 Grenoble Cedex, France

(Reçu le 18 mai 1976, révisé le 21 juin 1976, accepté le 5 juillet 1976)

\begin{abstract}
Résumé. - La forme du spectre de R.P.E. dû à un système avec un couplage vibronique $E \otimes \varepsilon$ est développée dans une série de puissances de $\omega^{-1}$ (où $\omega$ est la fréquence du champ électromagnétique). Une combinaison des coefficients de $\omega^{-1}$, s'ils étaient déterminés expérimentalement, pourrait tester si la coalescence des raies à température élevée est due au rétrécissement par mouvements ou non.

Abstract. - The Electron Spin Resonance spectral line-shape function of a vibronically coupled $E \otimes \varepsilon$ system is expanded as a series of powers of $\omega^{-1}(\omega$ is the frequency of the electromagnetic field). A combination of the coefficients of $\omega^{-r}$, when established experimentally, can test whether or not the high-temperature coalescence of lines is due to motional narrowing.
\end{abstract}

The Electron Spin Resonance (E.S.R.) spectrum arising from an orbital doublet in a cubic system, subject also to vibronic coupling, has been extensively researched $[1,2]$ for its dependencies on temperature, orientation of the magnetic field, the local strain distribution and, from a theoretical standpoint [3, 4], on various physical parameters.

In this note we draw attention to a further aspect of the E.S.R. spectrum, namely its dependence on the frequency of the absorbed electromagnetic radiation, with a view of deriving information on the appearance of high-temperature coalescence of anisotropic E.S.R. lines and the motional narrowing of the central line $[4,5]$. This phenomenon is simply described (and also widely found [6]) in the so-called dynamic limit of the $E \otimes \varepsilon$ coupling. The theory of this E.S.R. spectrum is primarily due to Ham. From its extended form (ref. [7] and equations (17)-(20) of ref. [4]) it emerges that at a chosen site $j$ the transition frequencies are :

$$
\begin{aligned}
h v_{ \pm} \equiv \hbar \omega_{0} \pm \hbar \Delta_{j} \\
=\beta H g_{1} \pm \frac{1}{2} \beta H q g_{2}\left[\left(3 n^{2}-1\right) \cos \varphi_{j}+\right. \\
\left.\quad+\sqrt{3}\left(l^{2}-m^{2}\right) \sin \varphi_{j}\right],
\end{aligned}
$$

due to the strain-split vibronic doublet having energies

$$
E_{ \pm} \equiv \pm \frac{1}{2} \delta_{j}= \pm q\left|V_{2}\right| e_{j}
$$

$\left({ }^{*}\right)$ On leave of absence from Soreq Nuclear Research Centre, Yavne, Israel. and

$$
h v_{\mathrm{A}}=\beta H g_{1}
$$

due to a singlet at an energy

$$
E_{\mathrm{A}}=3 \Gamma \text {. }
$$

These results are correct to $0\left(g_{2} / g_{1}\right)$ and neglect singletdoublet admixture (the pure dynamic case) and nuclear spins. $\beta$ is the Bohr magneton. $g_{1}$ and $g_{2}$ are the customary $g$-factors $[5,6], H$ and $(l, m, n)$ are the magnitude and direction cosines (with respect to a tetragonal axis) of the constant magnetic field, $e_{j}$ and $\varphi_{j}$ are the magnitude and angular parameter of the strain at the site $j, q$ is a reduction factor, and $V_{2}$ is the $E \otimes \varepsilon$ linear coupling constant. $3 \Gamma$ is related [3] to non-linear vibronic coupling.

To obtain theoretically the line-shape, we are required to introduce probabilities $p_{n}(n= \pm, \mathrm{A})$ of the system occupying any of the three vibronic states

$$
\sum_{n} p_{n}=1
$$

In systems to which this work is relevant

$$
\beta H g_{2}(\sim 0.1 \mathrm{~K})<\delta_{j}(\sim 1 \mathrm{~K})<3 \Gamma(\sim 30 \mathrm{~K}) .
$$

Then $g_{2}$ may be neglected in the probabilities $p_{n}$ which are functions of the temperature $T, \delta_{j}$ and $3 \Gamma$ alone.

We need the line-shape functions $I(\omega, H, j)$ which describe the absorption of radiation (of frequency $\omega$ ) 
at each site $j$ including also the effects of time dependent perturbations. These perturbations have the effect of :

I. A movement of the system between different spin-states of the same vibronic state.

II. A decay of the vibronic states if these arise from an excited electronic state.

III. Jumps of the system among the three vibronic states with the spin unchanged.

Effects I and II give rise to relaxation times $\tau_{n}(n= \pm, \mathrm{A})$. III may be described by a Markovian matrix [8]

where

$$
\Pi \equiv \Pi_{n m}
$$

$$
\sum_{m} \Pi_{n m}=0
$$

and in thermal equilibrium,

$$
\sum p_{n} \Pi_{n m}=0 \text {. }
$$

The combination of I and II with III is presumably small compared with either and is neglected here. Ham [9] found that at temperatures of the order of $10 \mathrm{~K} \Pi_{+_{-}}$and $\Pi_{-_{+}}$are dominated by a non-resonant Raman-process and are proportional to the square of the energy difference $\delta_{j}$ in (1).

We write

$$
\Delta \omega=\left(\omega-\omega_{0}\right) \mathbf{E}
$$

( $\mathbf{E}$ is the unit $3 \times 3$ matrix) and introduce the complex diagonal matrix

$$
\tau^{-1}=\left(\begin{array}{ccc}
\tau_{+}^{-1}-i \Delta & 0 & 0 \\
0 & \tau_{-}^{-1}+i \Delta & 0 \\
0 & 0 & \tau_{\mathrm{A}}^{-1}
\end{array}\right)
$$

as well as the vectors $\mathbf{p}=\left(p_{+}, p_{-}, p_{\mathrm{A}}\right)$ and

$$
\mathbf{1}=\left(\begin{array}{l}
1 \\
1 \\
1
\end{array}\right)
$$

An extension of the method of Abragam [8] yields at each site,

$$
I(\omega, H, j)=-I \operatorname{Im} \mathbf{p}_{j} \cdot\left[\Delta \omega+i\left(\Pi_{j}-\tau_{j}^{-1}\right)\right]^{-1} .1
$$

where $I$ is the integrated intensity due to a single site. The total intensity $I_{\mathrm{t}}$, being a sum over all sites $j$, is

$$
I_{\mathrm{t}}(\omega, H)=\sum_{j} I(\omega, H, j)
$$

When we expand $I_{t}$ for large frequencies,

$$
I_{t}(\omega, H)=\sum_{r=1,2, \ldots} I^{(r)} /(\Delta \omega)^{r} .
$$

We shall now estimate

$$
\begin{aligned}
R & \equiv \frac{I^{(6)}}{\left(I^{(2)}\right)^{2} I^{(4)}}= \\
& =\frac{\sum_{j} \mathbf{p}_{j} \cdot\left(\Pi_{j}-\tau_{j}^{-1}\right)^{5} \cdot 1}{I^{2}\left(\sum_{j} \mathbf{p}_{j} \cdot\left(\Pi_{j}-\tau_{j}^{-1}\right) .1\right)^{2} \sum_{j} \mathbf{p}_{j} \cdot\left(\Pi_{j}-\tau_{j}^{-1}\right)^{3} \cdot 1}
\end{aligned}
$$

and in particular its temperature variation.

As the temperature is raised to the region of motional narrowing (characteristically from $3 \mathrm{~K}$ to $30 \mathrm{~K}$, ref. [6]) some quantities (namely, $\tau$ and/or $\Pi$ ) will change by several orders of magnitude [10], others $\left(p_{ \pm}\right.$and $\left.p_{\mathrm{A}}\right)$ will vary slightly, and yet others $\left(g_{1}, g_{2}\right.$ and $\delta$ ) will remain nearly constant. We are interested in a rough estimate of $R$, but certain relations have to be borne in mind. Thus

$$
\begin{array}{ll}
\Pi \cdot 1=0 & \text { by (3) } \\
\text { p. } \Pi=0 & \text { by (4) }
\end{array}
$$

and the $j$-summation in (7) over odd powers of $\Delta_{j}$ vanishes (this last circumstance, consistent with, e.g. an angular independence of the strain distribution [3,4], achieves such simplifications in the sum (8) that only even powers $r$ remain, but is not crucial). When these relations are kept in mind $R$ becomes

$$
\begin{array}{r}
R=\sum \text { p. } \tau^{-1}\left(\Pi-\tau^{-1}\right)^{3} \tau^{-1} \cdot 1 / I^{2}\left(\sum \mathbf{p} \cdot \tau^{-1} \cdot 1\right)^{2} \times \\
\times \sum \text { p. } \tau\left(\Pi-\tau^{-1}\right) \tau^{-1} \cdot 1
\end{array}
$$

where we have omitted the index running over $j$ (the sites) from the quantities $\mathbf{p}$ (the occupation proba-

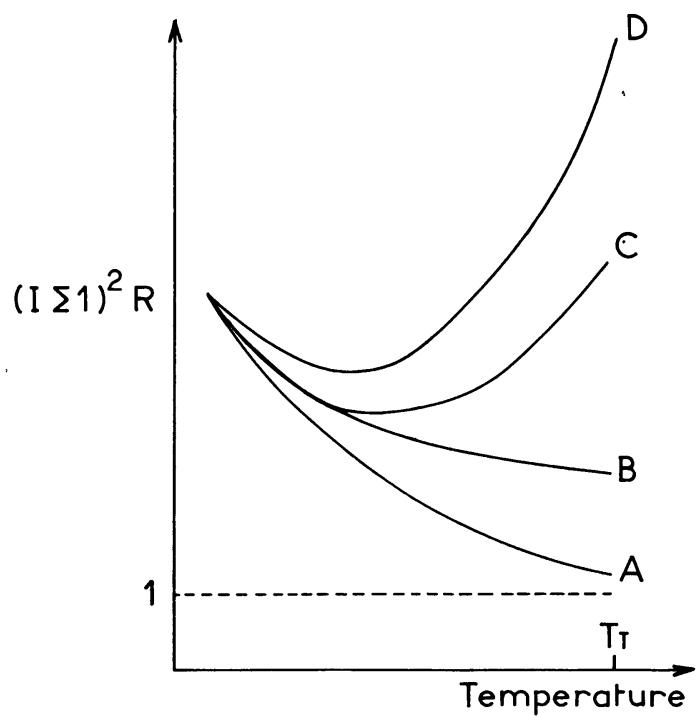

FIG. 1. - Schematic behaviour of equation (10) as a function of temperature. $T_{\mathrm{T}}$ is the temperature [6] at which essentially only the isotropic line (at $\omega_{0}$ ) appears. At this temperature the following possibilities may occur : Case $\mathrm{A}: \bar{\Delta}<\overline{\tau^{-1}}, \bar{\Pi}<\overline{\tau^{-1}}$, eq. (10) $\sim 1$. The disappearance of the anisotropic lines is not due to motional narrowing. Cases B, C and D : $\bar{\Pi}>\Delta, \bar{\Pi}>\bar{\tau}^{-1}$, eq. (10) $>1$.

Motional averaging and narrowing have taken place. 
bilities), $\tau^{-1}$ (the complex inverse relaxation time, eq. (5)) and $\Pi$ (the inter-state jump rate, eq. (3)). The above result can be written as

$$
\left(I \sum 1\right)^{2} R \sim 1+\bar{\Pi}^{2} \bar{\tau}^{2}+\bar{\Delta}^{2} \bar{\tau}^{2} .
$$

Here the squared factor on the left-hand side is essentially a temperature independent quantity which reflects the units of measurement. On the right-hand side, the barred quantities are appropriate site - and state - averages of $\Pi_{n m j} \tau_{n j}^{-1}$ and $\Delta_{j}$.

Figure 1 shows possible variations with rising temperature of $\left(I \sum 1\right)^{2} R$. Considering the situation that on the right-hand side extremity at $T_{\mathrm{T}}$ only the central line appears, the figure enables us to differentiate between two mechanisms which may explain the growth of the central line and the disappearance of the anisotropic lines. These are first (case A) thermal excitation of the singlet and a broadening of the doublet lines $\bar{\tau} \bar{\Delta} \rightarrow 0$ without any motional narrowing (i.e. $\bar{\Pi}<\bar{\Delta}$ ), and alternatively, cases $\mathrm{B}, \mathrm{C}$ or $\mathrm{D}$, where motional averaging and narrowing dominates broadening $\left(\bar{\Pi}>\bar{\tau}^{-1}\right)$ and its rate exceeds the low temperature width $\bar{\Delta}(\bar{\Pi}>\bar{\Delta})$.

In summary, we have shown that by analysing the far off-resonance regime of the E.S.R. spectrum as function of $\omega$, fairly clearcut information (equation (10)) on the motional narrowing mechanism can be obtained. This information has been wanting so far [4]. Admittedly, a frequency-dependent approach to E.S.R. is uncommon and difficult, and the asymptotic $\omega$-behaviour is even more so. However, one can operate at different frequencies and the coefficients of $(\Delta \omega)^{-r}$ can thereby be made accessible [11].

Acknowledgment. - I owe my thanks to Dr. F. Hartmann-Boutron for many interesting discussions.

\section{References}

[1] Chase, L. L., Phys. Rev. B 2 (1970) 2308.

[2] Schoenberg, A., Suss, J. T., LuZ, Z. and Low, W., Phys. Rev. B 9 (1974) 2047.

[3] Setser, G. G., Barksdale, A. O. and Estle, T. L., Phys. Rev. B 12 (1975) 4720.

[4] Reynolds, R. W. and Boatner, L. A., Phys. Rev. B 12 (1975) 4735.

[5] Watkins, G. D. and Ham, F. S., Phys. Rev. B 1 (1970) 4071.

[6] HaM, F. S. in Electron Paramagnetic Resonance, Ed. Geschwind, S. (New York, Plenum Press) 1972 ;

Hartmann-Boutron, F., J. Physique 29 (1968) 47.

[7] Herrington, J. R., Estle, T. L. and Boatner, L. A., Phys. Rev. B 3 (1971) 2933.

[8] Abragam, A. A., The Principles of Nuclear Magnetism (Oxford, Clarendon) 1961.

[9] Ham, F. S., Phys. Rev. 166 (1968) 307.
[10] Experimental relaxation rates on paramagnetic ions, e.g. on $\mathrm{Fe}^{3+}\left(\right.$ or $\mathrm{Co}^{2+}$ ), increase by 5 (or 8 ) orders of magnitude with a rise of temperature between $1.2 \mathrm{~K}$ and $4 \mathrm{~K}$ (or 3-25 K) probably due to a spin-flip process. [Articles by Pashinin, P. P. and Prokhorov, A. M. (or Zvervev, G. M. and Petelina, H. G.) in Spin-Lattice Relaxations in Ionic Solids, Ed. Manenkov, A. A. and Orbach, R. (Harper and Row, N. Y.) 1966].

[11] We have tried, but have been unable, to derive corresponding information from a moment analysis [12] on the spectrum, partly because of the inconvenience of having a Lorentzian line-shape. The supposition of Lorentzians is likely to be justifiable for the high frequency wings which is of interest to us. The low frequency side line-shape is discussed in ref. [8] (p. 53).

[12] Henry, C. H., Schnatterly, S. E. and Slichter, C. P., Phys. Rev. A 137 (1965) 583. 